\title{
Smartphone-based Walking Speed Estimation for Stroke Mitigation
}

\author{
Jeffrey Cox \\ Department of Computer \\ Science and Engineering \\ University of Tennessee at Chattanooga \\ Chattanooga, Tennessee, USA 37403
}

\author{
Yu Cao, Guanling Chen \\ Department of Computer Science \\ University of Massachusetts Lowell \\ Lowell, Massachusetts, USA 01854 \\ Email: ycao,glchen@cs.uml.edu
}

\author{
Jianbiao He \\ Department of Computer Science \\ Central South University, City of Changsha \\ Hunan Province, P.R. China 410012 \\ Email: jbhe@mail.csu.edu.cn
}

\author{
Degui Xiao \\ School of Computer Science \\ Hunan University, City of Changsha \\ Hunan Province, P.R. China 410012 \\ Email: dgxiao@hnu.edu.cn
}

\begin{abstract}
Each year, 15 million people suffer stroke worldwide. Among them, 5 million die and another 5 million are permanently disabled. Stroke recovery is a lifelong process. Clinical research have shown that gait velocity (a.k.a, walking speed) is a very powerful indicator of function and prognosis after stroke. In this paper, we focus on developing new algorithms to estimate the walking speed using pervasive devices, such as smartphone. While there are existing techniques to measure walking speed using inertial sensors, very little research has specifically involved smartphone, due to some unique challenges caused by pervasive devices, such as placement of the sensor and sensor drifting. We propose new practical algorithms based on high pass filter, integration of accelerator's reminder, and feedback loop. We evaluate our proposed approach with realworld data and present a through analysis on the results. The experimental results have indicated that proposed approach is a promising practical approach for gait speed estimation.
\end{abstract}

\section{INTRODUCTION}

Worldwide, 15 million people suffer stroke each year. Among them, 5 million die and another 5 million are permanently disabled [1]. Stroke recovery is a lifelong process. Stroke recovery usually begins with rehabilitation that aims to restore independence by improving physical, mental and emotional functions. Past clinical research have shown that gait velocity (a.k.a walking speed) is a very powerful indicator of function and prognosis after stroke [2][3]. Recent clinical research [4][5] also indicated that changes in velocitybased community ambulation can be correlated to clinically meaningful changes in stroke-related function and quality of life. Furthermore, the medical professionals could harness the gait velocity information to make more accurate diagnosis and provide better medical suggestions to improve the life of quality of the patients.

On the other hand, in the last decade, we have witnessed the field of information technology is a rapidly expanding with many different applications in biomedicine. In particular, many applications are being found in terms of allowing physician to collect physiology data for chronic conditions in natural living environments. This opens an unprecedented opportunity to discover early predictors and novel biomarkers to support clinical decision making and to reduce healthcare cost [6]. This is a significant cost cutter, as it relieves the necessity of having a health professional on hand to measure a patient's progress in what is often a slow recovery process.

The primary aim of this project is to use smartphone accelerometers to measure the walking speed (gait velocity) of users. Smartphone with built-in accelerometers and gyroscopes are becoming increasingly ubiquitous, and thus it is helpful to devise a method of measuring walking speed with them. By using a device that many users will already have, overall health care costs should be significantly reduced. While there are some on-going research efforts in walking speed estimation using inertial sensor [7][8], very little research has specifically involved smartphone. There are several unique challenges caused by pervasive devices. The first challenge arises as a result of the placement of the sensor. Authors in paper [9] examines the variation of precision of activity recognition based on the location of the sensor. The results of this paper indicate that while a waist-located sensor is not necessarily the ideal placement of a sensor for measuring walking, it is one of the better ones. The only location that had better precision for activity recognition of walking was the wrist, and most smartphones are too large to comfortably attach to the wrist. In our research, we use smartphone as a sensing device and it is unrealistic to wrap or attach the smartphone into the wrist. Therefore, the proposed approach could not be employed in our research. The second challenge is the drifting issue of the sensors used in smartphone, which means the output signal of the sensor slowly changes independent of the measured property. The drift issue is a greater challenge for smartphone because the accelerometers and gyroscopes in the smartphone are usually low-end sensors due to the cost considerations. In paper [10], the authors introduced a reasonable approach to producing a self-calibrating algorithm for sensor-based activity monitoring. This algorithm takes advantage of the fact that the acceleration due to gravity, $g$, is the only acceleration on the sensor when it is still. This fact can be used to calibrate the sensor. However, an obvious issue with this calibration algorithm is that it only works when the sensor is held still, which will obviously not be the case when the subject is 
walking. In this paper, we design a new mobile app to measure the patients' gait speed. This new app includes new practical algorithms to address aforementioned issues. Our proposed algorithms first employ a high pass filter to subtract the low frequency accelerations (such as gravity) from the raw sensor data. Then we give an initial estimation of the walking speed by integrating the reminder of the acceleration data. Finally, we develop a feedback loop-based approach to further refine the speed estimation. Our mobile app will remind the user to measure the gait velocity if the user forgot to do so. The proposed approach is validated with real-world data and the experiments have indicated that proposed approach is a promising practical approach for gait speed estimation.

\section{MotiVATIONS}

Walking speed is a good indicator of the general health of a stroke patient, and this project aims to provide physician with an easy way to measure that. Since most patients will already have a smartphone with an accelerometer built into it, physicians will not have to provide them with any special hardware to monitor, but will instead be able to simply provide them with an app. Smartphone also allow for easy upload of information to a database, as they usually have an internet connection. This project aims to make it easier to assess the health of patients by estimating walking speed without the necessity of specialized hardware.

From the technology point of view, the problem of walking speed estimation is interesting intellectually for two reasons. While other research has been done in using accelerometers to measure walking speed, very little of it has specifically involved smartphone. Using smartphone presents some extra challenges. When using a dedicated accelerometer, the issue of battery life can generally be ignored, however with smartphone it becomes one of the primary concerns. One of the challenges of this project was to produce an app that can measure the walking speed of participants without draining the battery of the phone too quickly. A larger issue with using smartphone is that the accelerometers used in smartphone may be of lower quality than some dedicated accelerometers. A final issue with smartphone is that both data storage and processing power are usually very limited, and thus one of the challenges was to create an algorithm that does not overtax the phone. In general, to not interfere with the normal function of the phone, the algorithm should not use more than $20 \%$ of the CPU and RAM. [11]

A greater challenge arises as a result of the placement of the accelerometer and sensor drifting. Low-quality accelerometers such as those in accelerometers experience a large amount of drift [10]. Other research in this area managed to solve this problem by placing the sensor at a location where at certain points the velocity is zero, known as the Zero Update Velocity Technique. However, for a waist placed sensor there is no point where the horizontal velocity is known to be a certain value, so there is not a point where the sensor can be recalibrated. Since the sensor in the iPhone experiences substantial drift in only a few meters, this issue must be addressed for any accurate estimate of walking speed to be achieved.

\section{RELATED WORK}

There is a substantial amount of work that has been done in this area. In this section, we list six papers in particular that provide a solid foundation for this research.

The paper "Inertial Sensor-Based Methods in Walking Speed Estimation: A Systematic Review"[12] in particular provides a good overview of the total work that has been done in the area. This paper looks at four different aspects of walking speed estimation: the sensor specifications, sensor attachment location, the design of the experiment, and the actual walking speed estimation algorithm. In terms of sensor specifications, two pieces of hardware were looked at, accelerometers and gyroscopes. For the accelerometers, specifications varied from $\pm 2 \mathrm{~g}$ to $\pm 50 \mathrm{~g}$. For gyroscopes, specifications varied from $\pm 150^{\circ}$ per second to $\pm 1000^{\circ}$ per second. The accelerometer and gyroscope used in this project had measurements ranges of $\pm 2 \mathrm{~g}$ and $\pm 1000^{\circ}$. According to the review, many different studies have been done using different locations of the sensors, with most using either thigh, shank or foot placements. Some of the studies used multiple sensors, however for this study we used a single sensor located at the base of the subject's spine. The review also examined the various experimental designs, mentioning two broad forms of experimentation: treadmill walking and overground walking at a known speed. For testing in this project, subjects were told to walk a known distance. The final aspect of walking speed estimation that this review looked at was the actual algorithm used in estimating the walking speed. According to the review, three main techniques were used, but we will only be using a variation of the third, direct integration, which the review claims was the most accurate of the three techniques. A more thorough description of this technique will be included in section 4 of this report.

A second paper that was very helpful in providing a background for this work is "Sensor Positioning for Activity Recognition Using Wearable Accelerometers." [13] This paper examines the variation of precision of activity recognition based on the location of the sensor. The results of this paper indicate that while a waist-located sensor is not necessarily the ideal placement of a sensor for detecting walking, it is one of the better ones. The only location that had better precision for activity recognition of walking was the wrist, and most smartphone are too large to comfortably attach to the wrist.

As mentioned in an earlier section of this paper, one of the challenges in using a pocket located sensor is accelerometer drift, which requires a constant recalibration of the sensor at regular intervals. Fortunately, the paper "A Real-Time and Self-Calibrating Algorithm Based on Triaxial Accelerometer Signals for the Detection of Human Posture and Activity" [14] contains a reasonable approach to producing a self-calibrating algorithm. This algorithm takes advantage of the fact that the acceleration due to gravity, $g$, is the only acceleration on the sensor when it is still. This fact can be used to calibrate the sensor. One obvious issue with this calibration algorithm is that it only works when the sensor is held still, which will obviously not be the case when the subject is walking. One of the goals of this project was to find a way to keep the sensor calibrated while the user is moving, possibly by performing an exact calibration at times when the user stops and then finding 
a way to approximate the calibration while the subject is in motion.

Recognizing when the user is walking is an important part of making this project practical. Ultimately, the goal is to have the phone collecting data continuously, but only analyze it while the subject is actually walking. Thus a way to identify activities is needed to make this project practical. In "Activity Recognition Using Cell Phone Accelerometers"[15] an algorithm is presented using cell phone accelerometers that correctly identifies when the user is walking approximately $93.6 \%$ of the time. This is accomplished by taking data collected from the accelerometers, and examining six different features of the data. The six features looked at were the average acceleration for each axis, the standard deviation for each axis, the average absolute difference for each axis, the average resultant acceleration, the time between peaks (for activities, like walking, which regularly repeat themselves) and a binned distribution of the range of values for each axis. The major challenge in implementing this algorithm will be to find a way to keep it from taxing the battery of the cell phone, as it is intended to run continuously. If the data mining prevents the device from operating without a recharge for a normal 16-hour day, then it is probably impractical for the typical user. [11]

In order to separate out the horizontal component of the acceleration of the accelerometer, the paper "Using Gravity to Estimate Accelerometer Orientation"[16] was extremely helpful. This paper presents a formula for extracting both the vertical and horizontal components of the acceleration from the overall accleration, by taking advantage of the fact that gravity is a constant force regardless of the reference frame. Specifically, taking the vector corresponding to gravity as $g=\left(g_{x}, g_{y}, g_{z}\right)$, the overall acceleration a as $a=\left(a_{x}, a_{y}, a_{z}\right)$, and the dynamic component of the acceleration as $d=\left(a_{x}-\right.$ $\left.g_{x}, a_{y}-g_{y}, a_{z}-v_{z}\right)$. Finally, the vertical component of the acceleration was found to be $v=\frac{((d \cdot g) * g}{(g \cdot g))}$ [16]. The horizontal component of the user's acceleration was thus: $h=d-v$. However, as the paper notes, there is no way to separate out the components of horizontal force, as there is no static acceleration to reference against.

Finally, the most relevant paper that we will draw on has to do the walking speed algorithm itself. The paper "Walking Speed Estimation Using a Shank-Mounted Inertial Measurement Unit" [17] presents an algorithm for measuring walking speed using a shank-mounted bi-axial accelerometer and a gyroscope. This was accomplished by assuming that the initial velocity at the start of each stride was zero, and then integrating the acceleration on both axes to find the velocity in both the $\mathrm{x}$ and $\mathrm{y}$ directions. From this, the stride length and stride velocity were found. In order to determine the start of a new stride, the shank vertical was tracked, and when it was straight it was considered the start of a new stride. For our research, we will use a similar algorithm, but will adjust it for a waist located triaxial accelerometer.

\section{PROPOSED APPROACH}

\section{A. System Design}

In our system, we use a fourth generation iPod Touch. Development was done in Objective-C using Xcode for extracting data from the iPod's sensors, and was then uploaded to a PC to be analyzed by a program written in Java. The primary sensor used in the iPod was the three-axis accelerometer. The accelerometer was polled approximately 60 times per second, as a greater polling rate resulted in greater noise in the data. To collect data, a program was written for the iPod which would begin collecting data five seconds after the user pushed the start button. The five second interval allowed the user to attach the iPod, which was in a case with a belt clip, to his/her belt at the base of the spine. We place the iPod at the base of the spine for two reasons. First, the lower back is a position where there is a relatively small amount of side-to-side motion. [13] This is important because there is no reference point for separating out the side to side motion from the forward motion. Secondly, this placement keeps one axis of the iPod pointed directly in the direction of the test subject's motion. For the iPod's reference frame, this was the z-axis. Thus, the other two axes could be effectively ignored, as virtually all forward motion took place along that axis.

\section{B. Data Analysis}

The first step of data analysis is the high pass filter. In order to find the acceleration due to gravity, we employ a high pass filter which subtracted the low frequency accelerations (such as gravity) from the raw sensor data. One issue arising from the use of a high pass filter is that in walking, some of the accelerations are sufficiently low frequency that they are eliminated by the filter. However, since this effect is related to the walking speed it can be accounted for in post-processing. The acceleration due to gravity was then used to determine the horizontal component of the acceleration, inspired by the method described in paper [16].

Next, we will first determine when the test subject began walking. It is assumed that before beginning walking they are relatively still, and so once a certain threshold of acceleration in the accelerometer data is reached (in this case, approximately $0.1 \mathrm{~g}$ ) it is assumed that the subject has begun walking. Prior to this point, an integration of the acceleration is done to ensure that the actual walking speed algorithm has an accurate starting speed.

Merely integrating the reminder of the acceleration data was found to have a serious problem. For a combination of reasons, not every deceleration was picked up by the sensor, and so the velocity was found to increase continuously over time. This is most likely a combination of accelerometer drift and the fact that some of the deceleration was likely eliminated by the high pass filter used to separate out the force of gravity. A further possibility is that the iPod's accelerometer may not be precise enough to accurately transition quickly between two sequential sharp accelerations in opposite directions.

Regardless of the cause, this error was found to be related directly to how fast the user walked, with some minor variation. The speed the user walked at is tied in to the overall sum of accelerations on the $\mathrm{z}$-axis, and this was used to make adjustments to the walking speed.

The basic principle in adjusting the speed is the application of a synthetic force applied as a final step in integration of the acceleration. The strength of this synthetic force general varied from between $1 \%$ and $3 \%$ of the current velocity at that point, determined by an adjustment value calculated as follows. 
The adjustment constant used to determine the synthetic force was based on the sum of the accelerations on the z-axis. This in turn was related to three factors: the total distance traveled, the speed of the subject, and the variation in the walking speed over the course of the test. For a distance of sixty meters, the total acceleration was found to vary between $2 \mathrm{~g}$ and $6 \mathrm{~g}$, increasing almost linearly with speed. This value was then found to vary linearly with distance, at least up to 130 meters. When the user walked with varying speeds during the test, the results were somewhat less predictable, though variation in walking speed usually resulted overall accelerations somewhat higher than a constant walking rate.

In order to remove the effect of distance, a feedback loop was inserted into the program. This loop estimated the distance a user walked by first assuming that the user walked at 1.5 $\mathrm{m} / \mathrm{s}$, and then using that value to obtain an adjustment to the walking speed calculation. This new value was then averaged with the original estimate and passed back in to the loop to find a new estimate for the walking speed. This was repeated until the estimate and the actual result were within $0.1 \mathrm{~m} / \mathrm{s}$ of each other. This approach worked quite well for most cases, but was found to break down for two boundary cases. For extremely high speeds, each pass through the filter resulted in estimates that were consistently too high. This was partially corrected by assuming that no test subject were walking faster than 2.2 $\mathrm{m} / \mathrm{s}$, which is reasonable considering that is very challenging for anyone to walk faster than that speed. The second boundary case that was problematic was the case for when the overall speed was fairly slow, but the variation in speed was large. For this case, the speed was underestimated, as the variation in walking speeds caused the feedback loop to overestimate the adjustment value, resulting in a lower estimation of walking speed than the actual speed. This was usually a minor effect, unless the variation was very high and the actual speed very low, in which case the algorithm produced faulty results.

In order to test the algorithm, subjects were asked to walk a known distance, either 66 or 132 meters, which are two representative distance used in therapy practice. The subjects' speed was calculated based on the length of time it took them to traverse this distance. This result was then compared to the estimate produced by the walking speed algorithm to find the percent error in the estimate.

\section{RESUlts}

Using the method described in the previous section results in a fairly accurate estimation of walking speed for distances between $60-130$ meters and for speeds between $0.8 \mathrm{~m} / \mathrm{s}$ and 2 $\mathrm{m} / \mathrm{s}$. For most cases, the error was less than 10 percent, though high speeds or for large variation in walking speed the error was sometimes higher.

18 tests were performed, nine on the primary test subject and then the other 9 were spread across six different test subjects. The primary test subject was a tall male, but other test subjects included a small child and 2 females. No correlation was found between related to either height or sex, but further testing is needed to confirm this.

The overall average percent error between the 18 tests was found to be approximately $6.8 \%$. The largest error on any single test $13.8 \%$, and approximately $25 \%$ of the tests had an error between $9 \%$ and $14 \%$.

TABLE I. RESULTS

\begin{tabular}{||c|c|c|c||}
\hline Subject & Actual Speed $(\mathrm{m} / \mathrm{s})$ & Calc. Speed $(\mathrm{m} / \mathrm{s})$ & \% Error \\
\hline 1 & 1.6 & 1.49 & 6.88 \\
\hline 1 & 1.13 & 1.14 & 0.88 \\
\hline 1 & 1.98 & 2.24 & 13.1 \\
\hline 1 & 1.04 & 1.14 & 9.62 \\
\hline 1 & 0.85 & 0.79 & 7.06 \\
\hline 1 & 1.64 & 1.48 & 9.76 \\
\hline 1 & 1.67 & 1.54 & 7.78 \\
\hline 1 & 1.07 & 1.15 & 7.48 \\
\hline 1 & 1.53 & 1.48 & 3.27 \\
\hline 2 & 1.42 & 1.58 & 11.27 \\
\hline 2 & 1.14 & 1.16 & 1.75 \\
\hline 3 & 1.46 & 1.28 & 12.3 \\
\hline 3 & 1.35 & 1.38 & 2.22 \\
\hline 4 & 1.63 & 1.54 & 5.52 \\
\hline 5 & 1.8 & 1.55 & 13.9 \\
\hline 5 & 1.37 & 1.34 & 2.19 \\
\hline 6 & 1.41 & 1.44 & 2.13 \\
\hline 7 & 1.44 & 1.52 & 5.52 \\
\hline
\end{tabular}

\section{CONCLUSIONS}

For the purpose of measuring the walking speed of stroke victims, this algorithm provides a practical and accurate measurement of walking speeds between $0.8 \mathrm{~m} / \mathrm{s}$ and 2.0 $\mathrm{m} / \mathrm{s}$ independent of gait. While further testing needs to be performed, especially on actual stroke victims, initial results indicate that unusual gait patterns do not effect this particular algorithm, as it is dependent solely on an integration of the acceleration, and does not use any aspect of the user's gait as a reference point. In general, physicians treating stroke victims do not need extremely precise estimates of walking speed, and thus this algorithms error margin of approximately $15 \%$ should not be a problem.

We envision the following future directions may further improve the performance. In particular, the feedback loop is not very precise, and a better way needs to be found to distinguish between high walking speeds and high variation in walking speed. One possible approach to this would be to analyze the data in smaller sections, as smaller sections of data would be less likely to have variation in walking speed.

A second improvement that could be done is a fine-tuning of the high-pass filter used to separate out the effects of gravity and determine the magnitude of the horizontal acceleration. A fine-tuning of this filter could improve the accuracy of this algorithm.

A third improvement is the placement of the smartphone. As it may be difficult to ask stroke patients to operate a smartphone and put it at the base of the spine, we will develop additional algorithms that will allow the sensor placement in other locations.

Additionally, as technology improves, it seems likely that more precise accelerometers will be included in phones. Given that this algorithm is almost directly related to the quality of the accelerometer, improvements in accelerometers should result in this algorithm producing more accurate results.

When compared with other algorithms that placed the sensor on either the shank or the foot, this algorithm produced 
slightly worse results. [18]. However, this may be offset by the more convenient waist location, as well as more accurate results for unusual gaits.

\section{ACKNOWLEDGMENT}

The authors would like to thank the anonymous reviewers for their valuable comments.

\section{REFERENCES}

[1] A. S. Go, D. Mozaffarian, V. L. Roger, E. J. Benjamin, J. D. Berry, W. B. Borden, D. M. Bravata, S. Dai, E. S. Ford, C. S. Fox, S. Franco, H. J. Fullerton, C. Gillespie, S. M. Hailpern, J. A. Heit, V. J. Howard, M. D. Huffman, B. M. Kissela, S. J. Kittner, D. T. L. J. H. Lichtman, L. D. Lisabeth, D. Magid, G. M. Marcus, A. Marelli, D. B. Matchar, D. K. McGuire, E. R. Mohler, C. S. Moy, M. E. Mussolino, G. Nichol, N. P. Paynter, P. J. Schreiner, P. D. Sorlie, J. Stein, T. N. Turan, S. S. Virani, N. D. Wong, D. Woo, and M. B. Turner, "Heart disease and stroke 2013 statistical update," Circulation Journal by American Heart Association, vol. 127, pp. 6-245, 2013.

[2] A. Schmid, P. W. Duncan, S. Studenski, S. M. Lai, L. Richards, S. Perera, and S. S. Wu, "Improvements in speed-based gait classifications are meaningful," Stroke, vol. 38, pp. 2096-2100, 2007.

[3] D. T. Wade, Measurement in Neurological Rehabilitation, ser. Oxford Medical Publications. Oxford University Press, USA, 1992.

[4] K. Salter, J. Jutai, L. Zettler, M. Moses, J. A. McClure, N. Foley, and R. Teasell, Outcome Measures in Stroke Rehabilitation. Error! Hyperlink reference not valid., address $=$ London, Ontario Canada, year $=2010$.

[5] R. Teasell, N. F. MSc, K. Salter, S. B. MSc, J. J. PhD, and M. S. $\mathrm{PhD}$, Evidence-Based Review of Stroke Rehabilitation (14th Edition). Canadian Stroke Network (CSN), 2011

[6] "Aaai spring symposia 2011 computational physiology," 2011.

[7] W. Khan, Y. Xiang, M. Aalsalem, and Q. Arshad, "Mobile phone sensing systems: A survey," 2012.

[8] S. Yang and Q. Li, "Inertial sensor-based methods in walking speed estimation: A systematic review," Sensors, vol. 12, no. 5, pp. 61026116, 2012.

[9] L. Atallah, B. Lo, R. King, and G.-Z. Yang, "Sensor positioning for activity recognition using wearable accelerometers," Biomedical Circuits and Systems, IEEE Transactions on, vol. 5, no. 4, pp. 320329, 2011.

[10] D. Curone, G. M. Bertolotti, A. Cristiani, E. L. Secco, and G. Magenes, "A real-time and self-calibrating algorithm based on triaxial accelerometer signals for the detection of human posture and activity," IEEE Transactions on Information Technology in Biomedicine, vol. 14, no. 4, pp. 1098-1105, 2010.

[11] J. Lockhart, G. Weiss, J. Xue, S. Gallagher, A. Grosner, and T. Pulickal, "Design considerations for the wisdm smart phone-based sensor mining architecture," in Proceedings of the Fifth International Workshop on Knowledge Discovery from Sensor Data. ACM, 2011, pp. 25-33.

[12] S. Yang and Q. Li, "Inertial sensor-based methods in walking speed estimation: A systematic review," Sensors, vol. 12, no. 5, pp. 6102 6116, 2012.

[13] L. Atallah, B. Lo, R. King, and G. Yang, "Sensor positioning for activity recognition using wearable accelerometers," Biomedical Circuits and Systems, IEEE Transactions on, vol. 5, no. 4, pp. 320-329, 2011.

[14] D. Curone, G. Bertolotti, A. Cristiani, E. Secco, and G. Magenes, "A real-time and self-calibrating algorithm based on triaxial accelerometer signals for the detection of human posture and activity," Information Technology in Biomedicine, IEEE Transactions on, vol. 14, no. 4, pp. 1098-1105, 2010.

[15] J. Kwapisz, G. Weiss, and S. Moore, "Activity recognition using cell phone accelerometers," ACM SIGKDD Explorations Newsletter, vol. 12, no. 2, pp. 74-82, 2011.

[16] D. Mizell, "Using gravity to estimate accelerometer orientation," in Proc. 7th IEEE Int. Symp. Wearable Computers (ISWC 2003). Citeseer, 2003.
[17] Q. Li, M. Young, V. Naing, and J. Donelan, "Walking speed estimation using a shank-mounted inertial measurement unit," Journal of Biomechanics, vol. 43, no. 8, pp. 1640-1643, 2010.

[18] A. Kose, A. Cereatti, and U. Croce, "Estimation of traversed distance in level walking using a single inertial measurement unit attached to the waist," in Engineering in Medicine and Biology Society, EMBC, 2011 Annual International Conference of the IEEE. IEEE, 2011, pp. $1125-1128$. 